\title{
Identifying the Prevalence of the Impostor Phenomenon Among Computer Science Students
}

\author{
Adam Rosenstein, Aishma Raghu, and Leo Porter \\ University of California, San Diego \\ La Jolla, California
}

\begin{abstract}
The Impostor Phenomenon (IP) is often discussed as a problem in the field of computer science, but there has yet to be an empirical study to establish its prevalence among CS students. One survey by the Blind app found that a high number of software engineers at some of the largest technology companies self-reported feelings of Impostor Syndrome; however, self-reporting of Impostor Syndrome is not the standard diagnostic for identifying whether an individual exhibits feelings of the Impostor Phenomenon. In this work, the established Clance IP Scale is used to identify the prevalence of IP among graduate and undergraduate computer science students at a large research-intensive North American institution. Among this population of over 200 students, $57 \%$ were found to exhibit frequent feelings of the Impostor Phenomenon with a larger fraction of women (71\%) experiencing frequent feelings of the Imposter Phenomenon than men (52\%). Additionally, IP was found to have greater prevalence among computer science students than among students of other populations from comparable studies. Due to the negative impacts associated with feelings of the Impostor Phenomenon, computer science education should work to improve student awareness and help student cope with these feelings.
\end{abstract}

\section{CCS CONCEPTS}

- Social and professional topics $\rightarrow$ Computing Education.

\section{KEYWORDS}

Impostor Phenomenon, Imposter Syndrome, Computing Education

\section{ACM Reference Format:}

Adam Rosenstein, Aishma Raghu, and Leo Porter. 2020. Identifying the Prevalence of the Impostor Phenomenon Among Computer Science Students. In The 51st ACM Technical Symposium on Computer Science Education (SIGCSE '20), March 11-14, 2020, Portland, OR, USA. ACM, New York, NY, USA, 7 pages. https://doi.org/10.1145/3328778.3366815

Permission to make digital or hard copies of all or part of this work for personal or classroom use is granted without fee provided that copies are not made or distributed for profit or commercial advantage and that copies bear this notice and the full citation on the first page. Copyrights for components of this work owned by others than ACM must be honored. Abstracting with credit is permitted. To copy otherwise, or republish, to post on servers or to redistribute to lists, requires prior specific permission and/or a fee. Request permissions from permissions@acm.org.

SIGCSE '20, March 11-14, 2020, Portland, OR, USA

(c) 2020 Association for Computing Machinery.

ACM ISBN 978-1-4503-6793-6/20/03 . \$15.00

https://doi.org/10.1145/3328778.3366815

\section{INTRODUCTION}

The Impostor Phenomenon (IP) ${ }^{1}$, as originally defined, is the experience of intellectual phoniness as perceived by high achieving individuals. These individuals have a great fear that others might discover that they are not as competent as they appear, attributing their successes to luck, knowing the right people, being in the right place at the right time, or even their personal charm [7].

Although there is a strong belief that the Impostor Phenomenon is more prevalent in computer science than in other fields, there has not yet been a carefully controlled study using a validated instrument to confirm this belief. In Blind's survey [18], 58\% of the 10,000+ software engineers self-reported feelings of Impostor Syndrome. However, self-reporting is not considered a validated means for measuring IP.

Historically, the Impostor Phenomenon is understood to be prevalent among underrepresented populations in a particular field; however, there is evidence that both underrepresented and represented groups are affected. The fact that more than half of the software engineers at large companies like Salesforce, Amazon, and LinkedIn reported feelings of IP suggests it extends beyond underrepresented groups in computer science.

It is widely acknowledged throughout computer science that the Imposter Phenomenon is prevalent among students [11]. Grace Hopper Celebration, an annual conference celebrating women in computing, has had panels and events which discuss the Impostor Phenomenon; however, most of them are purely anecdotal and do not address on a larger scale the roots in why the Imposter Phenomenon appears to be so prevalent within CS [9]. In sum, although it seems to be common knowledge that the Impostor Phenomenon exists among computer scientists, it has yet to be rigorously researched in computer science.

In this study, we use a validated metric of measuring the prevalence of IP, the Clance IP Scale. We used this scale in a survey of 203 computer science students at a large, public, research-intensive university to address the following questions: a) How prevalent is IP among CS students? b) What differences are there between sub-populations within CS? and c) How does the prevalence of IP in CS compare to other populations?

Both undergraduate and graduate students are surveyed, and their responses are analyzed. We find that more than half of the respondents meet the diagnostic criteria as frequently (or often) experiencing IP. We find that there is a significantly greater feeling of IP among CS students than published findings for college students in other fields and for health professional students. We find

\footnotetext{
${ }^{1}$ The terms Imposter Syndrome and Impostor Phenomenon are commonly used as synonyms, however we will use the term Imposter Phenomenon as this was the term first used in the seminal paper on the topic [6].
} 
no significant differences in the fraction of students who experience IP between undergraduate and graduate students or between racially underrepresented students and racially represented students. However, we find that a larger fraction of female students frequently experience feelings of IP than male students. We discuss implications of these findings and end with suggestions for future research on this topic.

\section{RELEVANT BACKGROUND WORK}

Given the lack of studies on the Impostor Phenomenon within the field of computer science, we provide a broader context of the history of, and studies pertaining to, IP. See also Sakulku for a more in depth review of the characteristics, suspected causes, and consequences of IP [25].

\subsection{A Brief History of the Impostor Phenomenon}

In 1978, Clance and Imes defined the term Impostor Phenomenon to designate an internal experience of intellectual phoniness in a select sample of high achieving women where, despite numerous achievements, the women did not experience an internal sense of success. These women persisted in believing that they were not really bright and had merely fooled anyone who thought otherwise [6].

Over the course of five years, the authors interacted with over 150 women who either earned doctoral degrees in various specialties, were respected professionals in their fields, or were students who had been recognized for their academic excellence. They found that despite quantifiable proof such as their earned degrees, scholastic honors, high achievement on standardized tests, praise and professional recognition from colleagues and respected authorities, these women did not experience an internal sense of success. They continued to believe that they were not intelligent and had fooled anyone who thought otherwise [6].

In a later study, Clance and O'Toole describe the features that accompany Impostor Phenomenon beliefs such as a dread of evaluation, fear of failure, guilt about success, and the interestingly reinforcing cycle of belief that the person must suffer in order to succeed and that self-doubt can be crucial to ensuring success [7].

Despite being initially studied as a women-centric experience with its causes and perpetuating circumstances being grounded firmly in the societal sex-role stereotypes [6], in the decade following the seminal work, research was conducted that found men experienced IP with just as much frequency as women. Topping's study found that among university faculty, male faculty members exhibited higher feelings of IP than their female counterparts [7, 27].

\subsection{Summary of IP Studies}

Table 1 provides a list of relevant studies of Imposter Phenomenon and the demographic of participants studied. For each reference, a checkmark denotes whether the study proposed a metric for diagnosing IP, a validation of such a metric, whether the study examined symptoms of IP, explored correlations of IP with other factors, and offered preventative measures. We expand on these topics in the following sections.

\subsection{Impostor Phenomenon Research and its Demographic Context}

Parkman summarizes the area in which the Impostor Phenomenon has been studied:

IP has been documented across the professions in a variety of industries including $\mathrm{K}-12$ education, health care, accountancy, finance, law, marketing, and higher education. [23]

As the majority of the research into IP has been done in the clinical or university settings, most studies find their participants amongst individuals participating in clinical therapy or university students [2, 5, 6, 8, 10, 12-16, 26, 28, 29].

Additional extensive research has been done in the fields of Gender Psychology [2, 5-7, 10, 12-16, 26-28], Health Professions and Medicine [14, 28], and Academia [2, 12, 13, 19, 22, 23, 29]. There has yet to be a quantitative study done that focuses on CS students.

\subsection{Effects of the Impostor Phenomenon}

There are a number of symptoms associated with IP; please see "Symptoms" column of Table 1 for associated studies. It is commonly accepted that feelings of IP contribute negatively to an individual's life, and several studies have shown that IP corresponds to increased levels in depression and anxiety [5, 8, 16, 23, 27].

Cokley et al. began exploring the relationship between perceived discrimination and mental health [17]. In a follow-up article, Cokley et al. continued their work by studying 322 educational psychology college students from a university in the Southwestern United States who self-identified as being part of minority groups. They questioned these students to understand their feelings of discrimination, levels of depression/anxiety, and impostor feelings [8].

Both of these papers had consistent findings and gained a better understanding of how the Impostor Phenomenon impacts students. The authors found that while African American students feel the highest levels of discrimination, Asian American students feel higher levels of the Impostor Phenomenon when compared to African Americans and Latino/a Americans. The predictive relationships were explored and it was surprisingly found that impostor feelings were a stronger factor in mental health than perceived discrimination and minority status stress for African Americans. Additionally, for Asian Americans, impostor feelings predicted increased levels of depression and anxiety [8].

This unfortunate result is not unique to minority students. Additional negative effects have been observed within other communities. For example, in the conclusions of their pilot study on impostor feelings amongst medical students, Villwock et al. stated:
Almost a quarter of male medical students and nearly half of female students experience [Impostor Phenom- enon] and [Impostor Phenomenon] was found to be significantly associated with burnout indices. Given the high psychological morbidity of these conditions, this association cannot be ignored. [28]

This motivates further studies to understand how to properly prevent burnout among students and working professionals. 
Table 1: Summary of Related Work

\begin{tabular}{|c|c|c|c|c|c|c|c|}
\hline \multirow{2}{*}{ Source } & \multirow{2}{*}{ Year } & \multirow{2}{*}{ Demographic } & \multicolumn{2}{|c|}{ Metric for Diagnosis } & \multirow{2}{*}{ Symptoms } & \multirow{2}{*}{$\begin{array}{c}\text { External } \\
\text { Correlations }\end{array}$} & \multirow{2}{*}{$\begin{array}{c}\text { Preventative } \\
\text { Measures }\end{array}$} \\
\hline & & & $\begin{array}{c}\text { Metric } \\
\text { Introduction }\end{array}$ & $\begin{array}{c}\text { Metric } \\
\text { Validation }\end{array}$ & & & \\
\hline [6] & 1978 & High-achieving Women & & & $\checkmark$ & & $\checkmark$ \\
\hline [27] & 1983 & University Faculty & & $\checkmark$ & $\checkmark$ & & \\
\hline$[7]$ & 1987 & Women & $\checkmark$ & & $\checkmark$ & $\checkmark$ & $\checkmark$ \\
\hline [10] & 1987 & Postgraduates \& Advanced Degree Holders & & $\checkmark$ & & & \\
\hline [16] & 1991 & Undergraduates in Intro. Psychology & $\checkmark$ & & $\checkmark$ & & \\
\hline [15] & 1993 & $\begin{array}{l}\text { Undergraduate Students \& } \\
\text { Clinical Clients }\end{array}$ & & $\checkmark$ & & & \\
\hline$[5]$ & 1995 & $\begin{array}{c}\text { Psychology Students \& } \\
\text { High-achieving Undergraduates }\end{array}$ & & $\checkmark$ & & & \\
\hline [14] & 1998 & Health Professional Students & & & & $\checkmark$ & \\
\hline [22] & 2001 & Middle School Students & & & & $\checkmark$ & \\
\hline$[12]$ & 2005 & Undergraduate Education Students & & & $\checkmark$ & $\checkmark$ & \\
\hline [30] & 2010 & Doctoral Students & & & & & $\checkmark$ \\
\hline [13] & 2011 & First Generation Doctoral Students & & & & $\checkmark$ & \\
\hline [23] & 2016 & Students, Faculty, and Staff & & & $\checkmark$ & $\checkmark$ & $\checkmark$ \\
\hline [28] & 2016 & American Medical Students & & & & $\checkmark$ & $\checkmark$ \\
\hline$[8]$ & 2017 & Minority Ed. Psych. Students & & & $\checkmark$ & $\checkmark$ & \\
\hline$[19]$ & 2017 & Professionals in Leadership Positions & & & $\checkmark$ & & \\
\hline [24] & 2017 & {$[\mathrm{~N} / \mathrm{A}]$} & & & & & $\checkmark$ \\
\hline$[2]$ & 2018 & $\begin{array}{l}\text { Undergraduate Business } \\
\text { \& Communication Students }\end{array}$ & & & & $\checkmark$ & \\
\hline$[4]$ & 2018 & Nursing Students & & & $\checkmark$ & & \\
\hline [26] & 2018 & STEM Doctoral Students & & $\checkmark$ & & & \\
\hline [29] & 2019 & $\begin{array}{l}\text { Undergrad Mgmt., Econ., Bus. Informatics, } \\
\text { Law, \& Program Eng. Students }\end{array}$ & & & & $\checkmark$ & \\
\hline
\end{tabular}

\subsection{Preventative \& Counteractive Measures}

There have been a number of studies on the ways to respond to and aid individuals with significant impostor feelings (see the last column of Table 1). In the initial study on IP [6], several psychotherapy responses are recommended for responses within therapy. At this point, the Impostor Phenomenon was thought to be a womencentric disorder, common among high-achieving women. Thus, a recommended therapy practice was to increase interaction with other high-achieving women through group therapy sessions. Here, the subject could speak with others-who might be struggling with a similar impostor belief-to gain a perspective on her thinking [6]. Alternatively, subjects could be aided in individual therapy sessions, although it often took several sessions for the subjects to exhibit signs of impostorism [6, 7].

Other work justifies that peer support groups provide help to graduate students who suffer from IP. The camaraderie of informal peer networks helps doctoral students to feel like they are not alone, and that there are others who have similar feelings of selfdoubt. These peer support groups were not introduced as formal meetings, rather it was the group dynamic of peers who were able to support each other with emails [30]. In Ramsey and Brown, several examples are given as to what academic libraries can do in order to help students "renegotiate their academic identities and overcome [the] Impostor [Phenomenon]". The theme of peer support groups is expanded upon by suggesting that campus entities should create workshops for students. A diverse campus staff is suggested to allow students to see themselves among those who they seek help and guidance from [24]. Although this work by Ramsey and Brown [24] focused on using libraries as the support network for students, both libraries and other campus entities could fulfill that role today.

The conclusions of Cokley et al. address several implications for university counseling centers and how they can incorporate this knowledge with the services that they provide [8]. They suggest that within initial assessment meetings, counseling centers should ask students about feelings of perceived fraudulence. It is possible to extend these suggestions and apply them to actions that could be taken by an educator as well.

With research evidence showing that the Impostor Phenomenon is so closely related to student mental health, college campuses have begun to take action to help ensure the well-being of students. The California Institute of Technology and the Massachusetts Institute of Technology include programs which help students identify impostor tendencies. Additionally, several other colleges have incorporated workshops regarding IP within their orientations [17]. By helping students define success, chronicle their achievements, and work to identify reasonable expectations, university departments have included students address this issue. Means for helping 
Table 2: Participant Characteristics

\begin{tabular}{|c|l|l|}
\hline Category & \multicolumn{1}{|c|}{ Subset } & Count (\% of total) \\
\hline \multirow{2}{*}{ Degree Level } & Undergraduate & $132(65 \%)$ \\
\cline { 2 - 3 } Gender & Graduate & $66(32.5 \%)$ \\
\cline { 2 - 3 } & Male & $149(73.4 \%)$ \\
\hline \multirow{2}{*}{$\begin{array}{c}\text { Represented } \\
\text { Status }\end{array}$} & $\begin{array}{l}\text { Non-Underrepresented } \\
\text { Minority }\end{array}$ & $173(85 \%)$ \\
\cline { 2 - 3 } & $\begin{array}{l}\text { Underrepresented } \\
\text { Minority }\end{array}$ & $25(12 \%)$ \\
\hline \multicolumn{2}{|c|}{ Total Participants } \\
\hline
\end{tabular}

* Refers to reported race/ethnicity that was not Asian or White.

† Some of the subset counts do not sum to the total number of valid responses due to the optional reporting of demographic information.

faculty and staff handle IP have also been incorporated. By identifying impostor tendencies during orientation and encouraging peer discussion groups, faculty and staff may better identify how to reduce these feelings. Ultimately these measures lead to a better environment for students, faculty, and staff [23].

\section{METHOD}

A survey containing the Clance IP Scale was administered to undergraduate and graduate students at a large public research-intensive North American institution. Three separate classes were selected for administration of the survey. These courses were chosen as they were popular upper-division or graduate courses with large enrollments to gather a larger sampling of advanced students. The undergraduate classes were an upper division Data Structures course which is typically comprised of 2 nd and 3 rd year students, and an upper division Natural Language Processing course which is comprised of mostly $3 \mathrm{rd}$ and 4 th year students. These were classes with large enrollments of undergraduate students who were computer science majors. Across the two classes, there were a total of 498 students enrolled. The graduate class selected was a graduate level class in Natural Language Processing. This popular course had among the largest enrollments in the graduate program with 190 students.

In accordance with our approved Human Subjects Board protocol, at each administration of the survey, a member of the research team entered the classroom, gave a brief introduction about the survey, then provided a web address for students to access the online survey through their personal devices. Students were not required to complete the survey and were not compensated for their participation. Also, students were not told about the nature of study at the time of recruitment; hence neither the terms "Imposter Phenomenon" nor "Imposter Syndrome" were used.

The survey consisted of two sections. The first section was the 20 -question Clance IP Scale ${ }^{2}$ (CIPS) that was originally printed in Pauline Rose Clance's The Imposter Phenomenon: Overcoming the Fear that Haunts your Success (1985). The scale is a 20 -item self

\footnotetext{
${ }^{2}$ The Clance IP scale is protected under copyright, and permission was obtained to use it in our study.
}

report scale where each item is rated on a Likert scale of 1 to 5 . A rating of 1 corresponds to "Not at all true" or total disagreement with the aforementioned statement and a rating of 5 corresponds to "Very true" or complete agreement. The items also measured the individuals dread of evaluation, fear of failure, guilt of success, and perception of others versus themselves [6]. CIPS has been reported to have strong internal consistency (Cronbach's $\alpha=0.92-0.96$ ) and strong validity $[5,15]$.

The second section of the survey consisted of requests for demographic information including gender, age, race/ethnicity, current degree program, and general questions about prior programming experience. Student responses were grouped by their corresponding demographic responses, and an ANOVA (Analysis of Variance) test was run to test for significance between different demographic groups. We define statistical significance as $p<0.05$.

A total of 203 valid responses were obtained from the 688 potential enrolled students (providing a $29.5 \%$ response rate). As seen in Table 2, of the total responses, $65 \%$ of the responses were from undergraduate students, $32.5 \%$ of the responses were from graduate students, and $2.5 \%$ declined to respond. Ages of participants were also collected on the following intervals: $17-21$ (52.2\%), 22-25 (40.4\%), 26-29 (5.9\%), 30-40 (<1\%), 50-60 (<1\%). $73.4 \%$ of the responses were from men and $26.1 \%$ of the responses were from women (1 participant did not respond). The races/ethnicities that were self-reported were Asian (76.3\%), White (8.9\%), Hispanic/Latino (7.1\%), Native Hawaiian/Other Pacific Islander (1.4\%), Middle Eastern $(<1 \%)$, and $2.5 \%$ declined to respond. Of the responses, $91.1 \%$ were from students majoring in CS.

\section{RESULTS}

Table 3 conveys CIPS results for the various demographics. Used here, ANOVA determines if there is a statistically significant difference between two or more means. This generalization of a $t$-test was used to compare demographic information. ANOVA provides no evidence to conclude that there is a statistically significant different in the means when comparing undergraduate and graduate students $(p=0.50)$, comparing age groups $(p=0.49)$, and when comparing racially represented (White/Asian) and racially underrepresented (not White/Asian) groups $(p=0.86)$. ANOVA found a statistically significant difference between male and female responses $(p=0.0046)$. The effect size between male and female responses, as measured by Cohen's $d$, is 0.47 . This is a small to medium effect size, where a small effect size is $d=0.2$ and a medium effect size is $d=0.5$.

\section{DISCUSSION}

\subsection{Interpreting Results}

Although Blind's study was based on a yes/no question rather than a validated diagnostic instrument, the findings of this paper support their finding that more than half of the individuals in computer science exhibit IP. Additionally, the data validates that the female students had significantly higher levels of impostor feelings than the male students. This may be attributed to the lack of representation of women in computer science, a topic that is commonly discussed at Grace Hopper Conferences. Furthermore, there was a lack of significant differences of IP between students 
Table 3: Survey Responses

\begin{tabular}{|l|l|l|l|}
\hline \multicolumn{1}{|c|}{ Population } & Count & \multicolumn{1}{|c|}{$\begin{array}{c}\text { CIPS } \\
\text { Mean (SD) }\end{array}$} & $\begin{array}{c}\% \text { meeting } \\
\text { diagnostic } \\
\text { criteria* }\end{array}$ \\
\hline All Valid Responses & 203 & $64.18(12.62)$ & $57.40 \%$ \\
\hline Undergraduate Students & 132 & $64.86(12.31)$ & $59.20 \%$ \\
\hline Graduate Students & 66 & $63.47(13.17)$ & $55.40 \%$ \\
\hline Male Students & 149 & $62.65(12.81)$ & $52.00 \%^{\ddagger}$ \\
\hline Female Students & 53 & $68.34(11.27)$ & $71.20 \%^{\ddagger}$ \\
\hline $\begin{array}{l}\text { Racially Represented } \\
\text { Student Groups }\end{array}$ & 173 & $64.01(12.69)$ & $60.50 \%$ \\
\hline $\begin{array}{l}\text { Racially Underrepresented } \\
\text { Student Groups }\end{array}$ & 25 & $64.48(13.18)$ & $56.90 \%$ \\
\hline
\end{tabular}

* "Met diagnostic criteria" refers to a CIPS score $\geq 62$

$\dagger$ Refers to reported race/ethnicity that was not Asian or White

* Cohen's $d=0.47$ and $p<0.005$

Table 4: CIPS Results From Other Studies

\begin{tabular}{|l|c|l|c|c|}
\hline Study & $N$ & \multicolumn{1}{|c|}{ Demographic } & $\begin{array}{c}\text { CIPS } \\
\text { Mean (SD) }\end{array}$ & $\begin{array}{c}\text { Estimated } \\
\% \text { meeting } \\
\text { diagnostic } \\
\text { criteria* }\end{array}$ \\
\hline$[14]$ & 477 & Health Professional students & $55.12(14.18)^{\sharp}$ & $31.38 \%$ \\
\hline$[12]$ & 124 & Education undergraduates & $54.24(13.27)^{\ddagger}$ & $27.93 \%$ \\
\hline$[8]$ & 322 & Minority Ed. Psych. students & $61.15(14.66)^{\dagger}$ & $47.69 \%$ \\
\hline$[29]$ & 169 & $\begin{array}{l}\text { Undergrad Mgmt., Econ., } \\
\text { Bus. Informatics, Law, } \\
\text { \& Program Eng. students }\end{array}$ & $58.50(12.16)^{\ddagger}$ & $38.67 \%$ \\
\hline
\end{tabular}

* Estimated CIPS score $\geq 62$, assuming scores are normally distributed ${ }^{\dagger} p<0.05$ when compared to CS students

$\sharp p<0.0001$ when compared to CS students

who identified as White/Asian and those who identified as members of under-represent groups. However, this could be due to our low sample sizes of underrepresented students in the dataset (including few Hispanic/Latino or African-American students).

We compared these results to findings for students using the same instrument in other fields. These prior findings are summarized in Table 4. Based on the CIPS score, we found that the results from this study reflect a statistically significant greater prevalence of IP among the sample of CS students than among 477 Health Professional Students [14], 124 Education college students in the Midwestern US [12], 322 Educational Psychology students from the Southwestern US who self-identified as minority students [8], and 169 undergraduate students majoring in Management, Economics, Business Informatics, Law, and Program Engineering from Russia [29] (using $t$-tests).

Although the CIPS mean was provided in these studies, the percentage meeting the diagnostic criteria was not. For this discussion, we also provide an estimate of the percentage who met the diagnostic criteria assuming the data was normally distributed (reconstructed using the reported mean and standard deviation) in Table 4. We assumed normality based on our data (using a KolmogorovSmirnov test, we found no evidence that our data was not normally distributed.) Overall, we were surprised by this finding that IP is more prevalent among CS students than students in other domains. This provides further motivation to understand what might cause IP to be more prevalent among CS students.

\subsection{Why IP is Prevalent in CS}

We have several hypotheses as to why more than half of students in computer science might experience frequent impostor feelings. One possible explanation may be the imbalance in access to K-12 computer science courses. Perhaps the absence of formal computer science education in many $\mathrm{K}-12$ education settings and that those schools with formal CS classes tend to be in higher income neighborhoods [21] leads to an uneven playing field in university-level $\mathrm{CS}$ courses. Given the presence of those with and without prior experience, it may seem to those without prior experience that those with prior experience have some kind of inherent knack (or geek gene [1] per-se) for computer science. Those without prior experience may believe that they do not have this perceived inherent ability and hence might feel like they do not possess the skill set for computer science.

Another possible explanation may be the message conveyed to students by their faculty. Lewis found that a prevalence (77\%) of computer science faculty believe that not everyone can succeed in CS [20]. If this belief is conveyed to students, it may cause anxiety in those students as to whether or not they have that inherent knack for computing.

Some students might also cause other students to doubt in their abilities, sometimes unintentionally or intentionally, by attempting to show off their knowledge in class. Barker et al. described this kind of behavior occurring in CS classes as distressing to other students by creating a defensive climate [3]. It is possible that defensive climates also lead to insecurities about student abilities. As that Barker et al. study also discussed that the defensive climate is particularly problematic for female students, that may also explain why women were more likely to suffer from imposter syndrome.

Yet another possible explanation is the nature of computer science content. When first learning computer science, learners are often presented with the idea of a black-box; there are parts that work, yet it is unnecessary to fully understand how. This could normalize not having a full understanding of the inner workings of a computer or programming language and that uncertainty might perpetuate a lack of confidence in students.

Lastly, individuals might have preconceived notions as to what type of person constitutes as a cultural fit within CS. This might lead to members of underrepresented groups feeling as if they do not belong regardless of their technical competence and academic abilities.

Each of these hypotheses require further study to understand the underlying reasons why a majority of students in CS suffer from IP and why there is a strong gender imbalance in this feeling of IP. Given the psychological strain IP places on students, it also behooves us to explore possible solutions (e.g., improve the inclusivity of the field so underrepresented groups feel more welcome).

\subsection{Threats to Validity}

By reviewing the context of this study we can acknowledge its potential limitations. The questions of the Clance IP scale ask about 
how much an individual relates to certain feelings. As a result, answers might vary slightly depending on when the survey was taken. Since our study was conducted during week 9 of a 10 -week term, increased stress levels could have influenced feelings of IP.

In addition, while there were 688 students enrolled in the courses, there were only a total of 203 valid responses that were acceptable for use in the analysis. This response rate of $29.5 \%$ might have been improved with compensation offerings in order to have increased participation. Additionally it seems possible that female students over-participated in the study, as $35 \%$ of study participants were female, yet roughly $18 \%$ of computer science majors at our institution are female.

All data collected was from students within a single department at a large public research-intensive university. It is possible that these results might not replicate when studied at other institutions.

An external threat to validity is that few comparable studies have been done within the previous 5 years regarding IP. Though this study reports that a majority of computer science students have frequent impostor feelings, it compares it to demographics from other fields based on prior literature that may no longer reflect present day numbers within those fields. For example, it is possible that the increased levels of impostor feelings are due to the generation of current students as opposed to being unique to the computing discipline.

\subsection{Personal Relevance}

While personal experiences were external motivators for the authors of this study, we were further encouraged regarding this study's relevance after a conversation with a research subject. Following one of the classes where a member of the research team administered the survey, a student approached the team member and inquired about the nature of the study. The undergraduate female student asked the member of the research team about the topic of the study, saying she strongly related to the content of the questions. Since the student had already completed the study and was curious about the research, we chose to disclose the study's topic. Once she heard about the Impostor Phenomenon, the student lit up; she expressed that she did not know that the feelings she had experienced as a student were commonly felt by others in the field. She was excited to hear that research was being done to understand how prevalent IP is within computer science, and thanked the authors for the work being done.

As with any quantitative research project, it is possible to lose sight of the individual students suffering detrimental effects from these feelings who need, and deserve, our encouragement and support. As a community, further research into IP could be highly beneficial for our students.

\subsection{Next Steps}

With the results from this paper, there are two potential paths for future research. The first is to further validate the claims of this paper by running the same study across multiple institutions around the world. As sample size increases and more diverse institutions are engaged, claims can be made with greater levels of confidence. An alternative route is to accept the conclusions of this paper and begin to explore why the Impostor Phenomenon is widespread throughout computer science. A possible next step would be a qualitative study interviewing computer science students to gain insight into what factors directly contribute to impostor feelings. Perhaps this would lead to preventative measures that we would be able to adopt for the benefit of our students. It also would be interesting to expand this study beyond students and work to identify IP levels among faculty members and professional software engineers.

Increased impostor feelings within computer science perhaps shed a light onto reasons for an unbalanced field with respect to diversity. Since impostor feelings are known to relate to increased levels in depression and anxiety [5, 8, 16, 23, 27], these feelings might be serving as a deterrent to students, thus preventing them from exploring computer science. Additionally, although there were no African-American students in this study's population, it is understood that their impostor feelings contribute more towards anxiety and depression than perceived racial discrimination [8]. Thus, it is imperative to work towards understanding how to mitigate these feelings.

As suggested by Henning et. al,

[I]t may ... be helpful to administer and discuss the ... CIPS measures to increase awareness of the dangers of ... IP. Furthermore evaluators of student performance should attend to any signs of these maladaptive traits and encourage more realistic perceptions through modelling, normalization of students' concerns and provision of specific feedback on faculty expectations of students. Finally, [associated] counselling centers ... may want to address ... IP through workshops, group treatments or brief individual therapy. [14]

As outlined in Section 2.5, several steps can be taken to mitigate impostor feelings. One such example is to provide group therapy sessions where high-achieving individuals have the opportunity to share their experiences as well as observe each others impostor feelings [6]. Alternatively, informal peer support groups allow for individuals to feel like they are not alone and that there are others who also feel self doubt [30]. Aside from professional counseling services, university departments can help spread awareness by including information about IP during orientation or through additional workshops. Conceivably, by increasing awareness and transparency surrounding IP, students will feel more comfortable and encouraged to either join computer science, or remain within the discipline.

\section{CONCLUSION}

After surveying over 200 computer science students at a large research-intensive North American institution, we found that $57.4 \%$ of them met the diagnostic criteria for the clinical range of the Impostor Phenomenon. Compared to prior findings in other disciplines, IP was found to be statically more prevalent in CS. In addition, this study found that there was a statistically significant difference between the populations of male students (52\%) and female students $(71.2 \%)$ in CS who reported feelings of IP. These results motivate the continued research of potential causes as to why this statistic is higher among computer science students than students in other disciplines and research on how to reduce feelings of IP among computer science students. 


\section{ACKNOWLEDGEMENTS}

Thank you to Professors Ndapa Nakashole and Paul Cao for their assistance with this study and to the reviewers for their helpful suggestions. We also thank Dr. Pauline Rose Clance for allowing us to use the Clance IP scale for this study. This work was supported in part by NSF award DUE-1712508.

\section{REFERENCES}

[1] Alireza Ahadi and Raymond Lister. 2013. Geek Genes, Prior Knowledge, Stumbling Points and Learning Edge Momentum: Parts of the One Elephant?. In Proceedings of the Ninth Annual International ACM Conference on International Computing Education Research. 123-128.

[2] Rebecca L. Badawy, Brooke A. Gazdag, Jeffrey R. Bentley, and Robyn L. Brouer 2018. Are all impostors created equal? Exploring gender differences in the impostor phenomenon-performance link. Personality and Individual Differences 131 (2018), 156-163.

[3] Lecia Jane Barker, Kathy Garvin-Doxas, and Michele Jackson. 2002. Defensive climate in the computer science classroom. In ACM SIGCSE Bulletin, Vol. 34. ACM, 43-47.

[4] Jennifer M Barrow. 2019. Impostorism: An evolutionary concept analysis. In Nursing forum, Vol. 54. Wiley Online Library, 127-136.

[5] Sabine M. Chrisman, W. A. Pieper, Pauline R. Clance, C. L. Holland, and Cheryl Glickauf-Hughes. 1995. Validation of the Clance Impostor Phenomenon Scale. Journal of Personality Assessment 65, 3 (1995), 456-467.

[6] Pauline Rose Clance and Suzanne Imes. 1978. The Imposter Phenomenon in High Achieving Women: Dynamics and Therapeutic Intervention. Psychotherapy: Theory, Research \& Practice 15, 3 (1978), 241-247.

[7] Pauline Rose Clance and Maureen Ann O'Toole. 1987. The Impostor Phenomenon Women \& Therapy 6, 3 (1987), 51-64.

[8] Kevin Cokley, Leann Smith, Donte Bernard, Ashley Hurst, Stacey Jackson, Steven Stone, Olufunke Awosogba, Chastity Saucer, Marlon Bailey, Davia Roberts, and et al. 2017. Impostor feelings as a moderator and mediator of the relationship between perceived discrimination and mental health among racial/ethnic minority college students. Journal of Counseling Psychology 64, 2 (March 2017), 141-154.

[9] Grace Hopper Organizing Committee. 2018. Grace Hopper 2018 Schedule. http://www.cvent.com/events/grace-hopper-celebration/ agenda-6083a0df738343e2ad8b262237e56423.aspx?p=13

[10] Patrick W. Edwards, Amos Zeichner, Norma Lawler, and Rachel Kowalski. 1987 A Validation Study of the Harvey Impostor Phenomenon Scale. Psychotherapy 24, 2 (1987), 256-259.

[11] Aidan L. Feldman and Matthew McCullough. 2014. Fighting Impostor Syndrome (Abstract Only). In Proceedings of the 45th ACM Technical Symposium on Computer Science Education. 728-728.

[12] Joseph R. Ferrari. 2005. Impostor Tendencies And Academic Dishonesty: Do They Cheat Their Way To Success? Social Behavior and Personality: an international journal 33, 1 (2005), 11-18.

[13] Susan K. Gardner and Karri A. Holley. 2011. "Those invisible barriers are real": The Progression of First-Generation Students Through Doctoral Education. Equity \&
Excellence in Education 44, 1 (2011), 77-92.

[14] Kris Henning, Sydney Ey, and Darlene Shaw. 1998. Perfectionism, the impostor phenomenon and psychological adjustment in medical, dental, nursing and pharmacy students. Medical Education 32, 5 (1998), 456-464.

[15] Sarah W. Holmes, Les Kertay, Lauren B. Adamson, C. L. Holland, and Pauline Rose Clance. 1993. Measuring the Impostor Phenomenon: A Comparison of Clance's IP Scale and Harvey's I-P Scale. Journal of Personality Assessment 60, 1 (1993), 48-59.

[16] John Kolligian Jr. and Robert J. Sternberg. 1991. Perceived Fraudulence in Young Adults: Is There an 'Imposter Syndrome'? fournal of Personality Assessment 56, 2 (1991), 308-326.

[17] Alicia Enciso Kevin Cokley, Shannon McClain and Mercedes Martinez. 2013. An examination of the impact of minority status stress and impostor feelings on the mental health of diverse ethnic minority students. Fournal of Multicultural Counseling and Development 41, 2 (2013), 82-95.

[18] Kim. 2018. 58 Percent of Tech Workers Feel Like Impostors. $\quad$ https://blog.teamblind.com/index.php/2018/09/05/ 58-percent-of-tech-workers-feel-like-impostors/

[19] Mona Leonhardt, Myriam N. Bechtoldt, and Sonja Rohrmann. 2017. All Impostors Aren't Alike - Differentiating the Impostor Phenomenon. Frontiers in Psychology 8 (2017).

[20] Clayton Lewis. 2007. Attitudes and Beliefs About Computer Science Among Students and Faculty. SIGCSE Bull. 39, 2 (June 2007), 37-41.

[21] Jane Margolis. 2010. Stuck in the shallow end: Education, race, and computing. MIT Press.

[22] Frank Pajares. 2001. Toward a Positive Psychology of Academic Motivation. The Journal of Educational Research 95, 1 (2001), 27-35.

[23] Anna Parkman. 2016. The Imposter Phenomenon in Higher Education: Incidence and Impact. Fournal of Higher Education Theory and Practice 16, 1 (2016), 51-60.

[24] Elizabeth Ramsey and Deana Brown. 2017. Feeling like a fraud: Helping students renegotiate their academic identities. College \& Undergraduate Libraries 25, 1 (2017), 86-90.

[25] Jaruwan Sakulku. 2011. The impostor phenomenon. The fournal of Behavioral Science 6, 1 (2011), 75-97.

[26] Marsha Simon and Youn-Jeng Choi. 2018. Using factor analysis to validate the Clance Impostor Phenomenon Scale in sample of science, technology, engineering and mathematics doctoral students. Personality and Individual Differences 121 (Jan. 2018), 173-175.

[27] Mary Elaine Harvey Topping. 1983. The Impostor Phenomenon: A Study of its Construct and Incidence in University Faculty Members. Ph.D. Dissertation. University of South Florida, Tampa, FL, USA. Advisor(s) Kimmel, Ellen B.

[28] Jennifer A. Villwock, Lindsay B. Sobin, Lindsey A. Koester, and Tucker M. Harris. 2016. Impostor syndrome and burnout among American medical students: a pilot study. International fournal of Medical Education 7 (2016), 364-369.

[29] Kenneth T. Wang, Marina S. Sheveleva, and Tatiana M. Permyakova. 2019. Imposter syndrome among Russian students: The link between perfectionism and psychological distress. Personality and Individual Differences 143 (2019), 1-6.

[30] Georgann Cope Watson and Andrea Smith Betts. 2010. Confronting otherness: An e-conversation between doctoral students Living with the Imposter Syndrome. Canadian fournal for New Scholars in Education 3, 1 (June 2010). 